\title{
Performance Analysis of Fuzzy Competitive Learning Algorithms for MR Image Segmentation
}

\author{
O. Mema Devi
}

Shahin Ara Begum

\author{
Department of Computer Science, Assam University, Silchar - 788011
}

\begin{abstract}
Neuro-fuzzy approach have attracted considerable attention in the computational intelligence and segmentation algorithms have been increasingly in developed in improving the accuracy of medical diagnosis. Fuzzy set attempts to represent the human perception whereas neural network attempt to emulate the architecture and information representation scheme of human brain. In this paper a comparative study on the performance of the FCM and the variant fuzzy competitive learning algorithms including the generalized Kohonen's competitive learning (GKCL)-based algorithms (KCL, fuzzy KCL (FKCL), fuzzy soft KCL (FSKCL)) and the learning vector quantization (LVQ)-based algorithms (LVQ, fuzzy LVQ (FLVQ), fuzzy soft LVQ (FSLVQ)) for MR image segmentation is presented. The performance of the algorithms are evaluated using the standard image quality indices such as MSE (mean squared error) and IQI (image quality index) and the results indicate that the soft versions of fuzzy competitive learning algorithms produces more promising results and require less CPU time than the other learning algorithms. Further, the LVQ-based algorithms have better performance according to the values of MSE and IQI as compared to the KCL based algorithms and the FCM algorithm.
\end{abstract}

\section{Keywords}

MR image segmentation, fuzzy set, KCL, LVQ.

\section{INTRODUCTION}

Model selections for medical diagnosis and prognosis have been increasingly in attention in improving the accuracy of medical diagnosis. Model-based fault diagnosis methods based on fuzzy clustering, SOM and neural gas network attempt in grouping image pixels based on the similarity of their intensity profile in time. Fuzzy set and neural net models and the integration of these two paradigms for medical diagnosis and prognosis have been increasingly studied for many years. To extract the interested structures in MR images, many researchers aim to develop segmentation techniques including fuzzy logic, neural network and the integration of these two paradigms thus enhancing their individual capabilities.

MRI is a medical imaging technique that uses nuclear magnetic resonance of protons to produce proton density images [1] [2]. The image pixel value can be considered as subsets of parameters including the time constants characterization T1 (magnetization vector along with longitudinal axis) and T2 (transverse component) and proton density (that has distinct value). Another MRI scan is diffusion MRI and it measures the diffusion of water molecules in biological tissues. By changing the effect of these meters, MR images can differentiate the structures obtained from the same anatomical positions. A widely and successfully used neural paradigm for finding prototypes is the self organizing map (SOM). Concerning the major problems of self organizing map (SOM), the generalized Kohonen's competitive learning (GKCL)-based algorithms (KCL, fuzzy KCL (FKCL), fuzzy soft KCL (FSKCL)) and the learning vector quantization (LVQ)-based algorithms (LVQ, fuzzy LVQ (FLVQ), fuzzy soft LVQ (FSLVQ)) have been developed to improve performance and usability [3-6]. The $\mathrm{KCL}$ is a sequemtial type and the LVQ is a batch type. LVQ is a simplest case for self organizing map (SOM). Mingoti $e t$ $a l$. , [7] present a comparison of SOM neural network, fuzzy $c$ mean, $k$-means and hierarchical clustering algorithms. Based on the comparison it is found that fuzzy $c$ - mean clustering algorithm performed well in all situations than any other algorithms. LVQ attempts to update only the winning prototype, generalization of LVQ-fuzzy variant is developed to updates all the $c$-prototypes with the learning rule. Lin $e t$ al., [4] proposed a generalized Kohonen's competitive learning (GKCL) for MR image segmentation called fuzzy KCL (FKCL) and fuzzy soft KCL (FSKCL) and is successfully applied to two actual ophthalmology cases. Amongst the GKCL-based algorithms, FSKCL is the most robust to outlying lesions and can easily interfered with a biased set of learning rates. However GKCL algorithm has some limitations as they are highly sensitive to the MRI data set used and may affect the number of iteration. Fuzzy learning vector quantization (FLVQ) can reduce this sensitivity of parameters. It provides a subsequent link between batch FCM and LVQ and also overcomes the problems in LVQ. This was first discussed by Huntsberger and Ajjimarangsee [8]. The FLVQ is more suitable than GKCL-based algorithms in comparison and gained a successful batch clustering algorithm that is applied in MRI. Further, another modified batch clustering learning method called fuzzy-soft learning vector quantization (FSLVQ) is proposed in $\mathrm{Wu}$ et al., [4] and produce better performance than the FLVQ in comparison but it is tested with numerical data only. Based on this, Yang et al., [5] proposed the FSLVQ segmentation technique with MRI and it works well on Alzheimer disease (AD) MRI. The tested results of the FSLVQ are compared with the other LVQ-based and GKCLbased algorithms and the comparison found that the FSLVQ is more robust and suitable. This paper focuses on the MR image segmentation techniques using fuzzy learning algorithms that have better detection of abnormal tissues and compare the fuzzy learning strategies of clustering algorithms that have been considered fuzzy according to the definitions found in the existing literatures. For the comparison of the algorithms we have downloaded the Mets Brain MR images from the website (January, 2012), http://myweb.msoe.edu/ martynsc/images/mri/mri.html.

The rest of the paper is structured as follows: section 2 provides the background on fuzzy image segmentation. Section 3 describes the KCL-based segmentation techniques for MR image, in section 4; the LVQ-based segmentation technique is described. Section 5 presents the image quality 
indices used to evaluate the different algorithms. The experimental results are discussed in section 6. Finally section 7 concludes the paper.

\section{FUZZY IMAGE SEGMENTATION}

Development of the methods for fuzzy image segmentation is constantly increasing for medical diagnosis and prognosis. Fuzzy set theory provides a number of suitable properties for pattern recognition diagnostic system due to its ability to deal with uncertainties, vagueness and incompleteness in medical diagnosis and prognosis. It can be used to represent fuzzy objects (both linguistic and/or set of variables) and fuzzy logic (reasoning methods). Torres et al., [9] presents a review on the current applications of fuzzy logic in medicine and bioinformatics. The main reasons for the application of fuzzy set theory in pattern recognition are: (i) its way of representation in linguistic approach with excellent formulation of input feature, (ii) representation of missing or incomplete knowledge as a degree of membership and (iii) its capability of drawing approximate inferences. Fuzzy set theory help to transfer a qualitative evaluation of the medical data into the algorithmic structure. Baraldi et al., [10], [11] present a survey on fuzzy clustering algorithms for pattern recognition. Literature survey shows that many fuzzy clustering algorithms aim to model fuzzy (i.e., ambiguous) unsupervised (unlabeled) patterns efficiently and is widely used for segmentation of MRI in brain tissue.

The concepts of fuzzy set are incorporated in the $c$-means framework to develop the FCM (fuzzy $c$-means). Let $X=\left\{x_{1}\right.$, $\left.x_{2} \ldots x_{n}\right\}$ be a data set and let $c$ be a positive integer greater than one. A fuzzy pseudopartition or fuzzy $c$-partition of $X$ is a family of fuzzy subsets of $X$, denoted by $P=\left\{\mu_{1}, \mu_{2} \ldots \mu_{c}\right\}$, which satisfies:

$\sum_{i=1}^{c} \mu_{i}\left(x_{j}\right)=1 \quad \forall x_{j} \in X$

The FCM minimized the following objective function:

$J_{F C M}=\sum_{i=1}^{c} \sum_{j=1}^{n} \mu_{i j}{ }^{m}\|x j-v i\|^{2}$

The parameter $m$ is a weight that determines the degree to which partial members of a cluster affect the clustering result. The FCM clustering algorithm is iteration through the necessary conditions for minimizing $J_{F C M}$ with the following update equations:

$$
v i=\frac{\sum_{j=1}^{n} \mu_{i j}{ }^{m} x_{j}}{\sum_{j=1}^{n} \mu_{i j}{ }^{m}}, \quad i=1, \ldots, c
$$

and

$$
\mu_{i j}=\left(\sum_{k=1}^{c} \frac{\left\|x_{j}-v_{i}\right\|^{\frac{2}{m-1}}}{\left\|x_{j}-v_{k}\right\|^{\frac{2}{m-1}}}\right)^{-1}, i=1 \ldots, c ; j=1, \ldots, n
$$

The algorithm proceeds as in $c$-means, along with the incorporation of membership. The algorithm is based on the assumptions that the desired number of clusters $c$ is given and in addition, a particular distance, a real number $m \in(1, \infty)$, and a small positive number $\varepsilon$, serving as the stopping criterion, are chosen. The process stops when the centroid is stabilize. That is, the centroids of the previous iteration are identical to those generated in the current iteration. Thus, the FCM algorithm is as follows [12-14]:

S1: Fix $m>1$ and $2 \leq c \leq n-1$ and give $k$ initial cluster centers $v_{i}$.

REPEAT

S2: Compute $\mu_{i j}$ with $v_{i}$ by eq. (4).

S3: Compute the objective function using equation (2).

S4: Update $v_{i}$ with $\mu_{i j}$ by eq. (3).

UNTIL (cluster centers stabilized).

The number of iteration taken by the algorithm depends on the position of the initial cluster prototypes. For random initialization of cluster prototype the algorithm converge to the desired results in less number of iterations if the initial cluster prototypes lies near the actual ones. Otherwise, the algorithm takes more iteration to find the actual prototypes. Thus, FCM clustering algorithm is sensitive to position of initial cluster prototypes. So, computational complexity is higher in FCM for a bad prototype initialization. Developments of the variations of the FCM algorithm are constantly increasing to perform well with medical data sets.

\section{KCL-BASED IMAGE SEGMENTATION}

In the KCL-based image segmentation techniques, SOM is used as the learning method for MR image segmentation and in this section we present a brief outline on three KCLbased algorithms viz., KCL, FKCL and the FSKCL algorithms. Although KCL network is originally not a clustering method, it could be used as a prototype generation algorithm called a LVQ. The FKCL and the FSKCL algorithms is the generalized KCL algorithm that is proposed by Karen Chia-Ren Lin et al., [4].

The KCL is based on the following objective function of the least squared error:

$$
L_{c}=\sum_{i=1}^{c} \sum_{j=1}^{n} \mu_{i j}{ }^{m}\left\|x_{j}-v_{i}\right\|^{2}
$$

Where

$$
\mu_{i j}= \begin{cases}1 & \text { if } \quad x_{j} \in \mu_{i} \\ 0 & \text { otherwise }\end{cases}
$$
data $x_{j}$ :

The KCL uses the following learning rule for one input

$v_{i}(t)=\mathrm{v}_{\mathrm{i}}(\mathrm{t}-1)+\alpha(\mathrm{t}) \mathrm{h}_{\mathrm{ij}}(\mathrm{t})\left(\mathrm{x}_{\mathrm{j}}-\mathrm{v}_{\mathrm{i}}(\mathrm{t}-1)\right)$

With

$h_{i j}(t)=$

$\left\{\begin{array}{c}1 \text { if }\left\|x_{j}-v_{i}(t-1)\right\|=\min _{1 \leq k \leq c}\left\|x_{j}-v_{i}(t-1)\right\| \\ 0 \quad \text { otherwise }\end{array}\right.$

Where $\alpha_{i}(t)$ is the learning rate of the node $i$ and is a monotonically decreasing function to $t, h_{i j}$ denotes the degree of excitation of the neuron $i$. Thus the KCL algorithm is as follows: 
S1: Fix $c, T$ and give $\varepsilon>0$.

S2: Initialize the weights $v_{i}(0)$ and the learning rate $\alpha(0)$. Set the iteration counter $t=1$.

S3: For $t=1,2, \ldots, T$;

$\alpha(t)=\alpha(0)\left(1-\frac{t}{T}\right)$

For $j=1,2, \ldots, n$;

Find $h_{i j}$ using eq. (7).

Update the winner $v_{i}(t)$ using eq. (6).

Obtain the objective function using eq. (5).

Next $j$.

S4: Compute

$E_{t}=\|v(t)-v(t-1)\|=\sum_{i=1}^{c}\left\|v_{i}(t)-v_{i}(t-1)\right\|$

S5: If $E_{t} \leq \varepsilon$, STOP; ELSE next $t$

In the FKCL algorithm the above KCL is extended by adding the FCM clustering. The FKCL uses the following learning rule for one input data $x_{j}$ :

$$
\begin{aligned}
& v_{i}(t)=\mathrm{v}_{\mathrm{i}}(\mathrm{t}-1)+\alpha(\mathrm{t}) \mathrm{h}_{\mathrm{ij}}(\mathrm{t})\left(\mathrm{x}_{\mathrm{j}}-\mathrm{v}_{\mathrm{i}}(\mathrm{t}-1)\right) \\
& \text { With } \quad \alpha(t) h_{i j}(t)=\mu_{\mathrm{ij}}^{\mathrm{m}(\mathrm{t})}
\end{aligned}
$$

Where $\mu_{i j}$ is the FCM membership, that is in the eq. (4). Thus the FKCL algorithm enables us to set up the index $m$ of fuzziness with the learning behaviour upon $t$. Thus the FKCL algorithm is as follows:

S1: Fix $c, T$ and give $\varepsilon>0$, fix $m_{0}$ and $m_{f}$ with $1.1<m_{f}<m_{0}<7$ S2: Initialize the weights $v_{i}(0)$ and learning rate $\alpha_{i}(0)$ of each node $i, i=1,2, \ldots, c$

$\mathrm{S} 3$ : For $\mathrm{t}=1,2, \ldots, \mathrm{T}$

$m(t)=m(0)+t\left(\frac{m_{f}-m_{0}}{T}\right)$

For $j=1,2, \ldots, n$;

Find $\mu_{i j}{ }^{m(t)}$ using equation (4).

Update all nodes $v_{i}(t), i=1, \ldots, c$ using the eq. (8) and eq.

Obtain the objective function using eq. (2).

Next $j$

S4: Compute

$$
E_{t}=\|v(t)-v(t-1)\|=\sum_{i=1}^{c}\left\|v_{i}(t)-v_{i}(t-1)\right\|
$$

S5: If $E_{t} \leq \varepsilon$, STOP; ELSE next $t$

In the FKCL algorithm, the learning rate and the degree of neuron excitation are put together with $\mu_{i j}{ }^{m(t)}$. In the FSKCL algorithm the FKCL algorithm is modified by keeping separately approximated using FCM membership functions $\mu_{i j}$ The FSKCL uses the following learning rule for one input data $x_{j}$ :

$$
v_{i}(t)=v_{i}(t-1)+\alpha(t) h_{i j}(t)\left(\mathrm{x}_{\mathrm{j}}-\mathrm{v}_{\mathrm{i}}(\mathrm{t}-1)\right)
$$

Where

$$
h_{i j}(t)=\left[\frac{\mu_{i j}}{\min _{1 \leq i \leq c} \mu_{i j}}\right]^{1+\frac{f(t)}{c}}, \quad \mathrm{i}=1, \ldots, c
$$

Where $\mu_{i j}$ is the FCM membership that is in the equation (3) and $f(t)$ is a positive strict monotonic increasing function of $t$ which controls the degree of neuron excitation. The learning rate in the FSKCL algorithm is as follows:

$$
\alpha_{i}(t)=\frac{\alpha_{0}}{\left(\frac{\alpha_{0}}{\alpha_{i}(t-1)}\right)+h_{i j}(t)}
$$

Thus the FSKCL algorithm is as follows:

S1: Fix $c, T$ and give $\varepsilon>0$

S2: Initialize the weights $v_{i}(0)$ and learning rate $\alpha(0)=\alpha_{\mathrm{i}}(0)=1$ for $i=1,2, \ldots, c$

S3: For $t=1,2, \ldots, \mathrm{T}$;

For $j=1,2, \ldots, n$;

Find $\mu_{i j}$ using eq. (4).

Find $h_{i j}(t)$ using the eq. (11)

Find $\alpha_{i}(t)$ using the eq. (12)

Update all nodes using eq. (10)

Obtain the objective function using eq. (2).

Next $j$

S4: Compute

$$
E_{t}=\|v(t)-v(t-1)\|=\sum_{i=1}^{c}\left\|v_{i}(t)-v_{i}(t-1)\right\|
$$

S5: If $E_{t} \leq \varepsilon$, STOP; ELSE next $t$

\section{LVQ-BASED IMAGE SEGMENTATION}

The batch type LVQ segmentation algorithms was started to overcome the sensitivity of parameters. In LVQ-based image segmentation the nodes are updated when each data is input in sequential type. However, for a given and fixed feature vector set, a batch version of the SOM can be considered so that it can be faster and does not require specification of any learning rate $\alpha_{i}(t)$. The LVQ algorithm uses the following learning rule for one input data $x_{j}$ :

$$
v_{i}(t)=\frac{\sum_{j} h_{i j} x_{j}}{\sum_{j} h_{i j}}
$$

Where

$$
\begin{aligned}
& h_{i j}(t)= \\
& \left\{\begin{array}{l}
1 \\
0
\end{array} \text { if }\left\|x_{j}-v_{i}(t-1)\right\|=\min _{1 \leq k \leq c}\left\|x_{j}-v_{k}(t-1)\right\|\right. \\
& \text { otherwise }
\end{aligned}
$$

Thus the batch LVQ algorithm is as follows:

S1: Fix $c, T$ and give $\varepsilon>0$.

S2: Initialize the weights $v_{i}(0)$. Set the iteration counter $t=1$.

S3: For $t=1,2, \ldots, T$;

Find $h_{i j}$ at time according to $v_{i}(t-1)$ using eq. (14)

Calculate the winner $v_{i}(t)$ using eq. (13).

Obtain the objective function using eq. (5)

Next $j$

S4: Compute 


$$
E_{t}=\|v(t)-v(t-1)\|=\sum_{i=1}^{c}\left\|v_{i}(t)-v_{i}(t-1)\right\|
$$

\section{S5: If $E_{t} \leq \varepsilon$, STOP; ELSE Next $t$}

The LVQ algorithm is extended in the FLVQ and FSLVQ algorithms by extending the fuzzy membership function. Based on the FCM membership function, the neural lateral interaction function in FLVQ is defined as follows:

$h_{i j}=\left(\mu_{i j}\right)^{m_{t}}=\left(\sum_{k=1}^{c} \frac{\left\|x_{j}-v_{i}\right\| \frac{2}{\left(m_{t}-1\right)}}{\left\|x_{j}-v_{k}\right\| \frac{2}{\left(m_{t}-1\right)}}\right)^{-m_{t}}$

Where

$m_{t}=m_{0}+t\left[\frac{\left(m_{f}-m_{0}\right)}{\max N I}\right]$

and the prototype update equation is:

$$
v i=\frac{\sum_{j}\left(\mu_{i j}\right)^{m_{t}} x j}{\sum_{j}\left(\mu_{i j}\right)^{m_{t}}}=\frac{\sum_{j} h_{i j} x_{j}}{\sum_{j} h_{i j}}
$$

Thus the FLVQ algorithm is as follows:

\section{S1: Fix $c, T$ and give $\varepsilon>0$}

S2: Initialize the weights $v_{i}(0), \mathrm{i}=1, \ldots, c$. Specify $m_{0}, m_{f}$ and maxNI.

Input all feature vectors $x_{j}, j=1, \ldots, n$

Set the iteration counter $t=1$

S3: For $t=1,2, \ldots, T$;

Calculate $m_{t}$ using equation (16)

Calculate all $h_{i j}$ at time $t$ according to $v_{i}(t-1)$ and $m_{t}$ using equation (15)

Calculate $v_{i}(t)$ using equation (17)

S4: Compute

$$
E_{t}=\|v(t)-v(t-1)\|=\sum_{i=1}^{c}\left\|v_{i}(t)-v_{i}(t-1)\right\|
$$

\section{S5: If $E_{t} \leq \varepsilon, \mathrm{STOP} ;$ ELSE next $t$ GOTO S3}

In the literature the parameter values suggested is $1.1<m_{f} \leq 7$ and in the special case of $m_{0}=m_{f}=m$, the FLVQ is identical to the FCM clustering algorithm. If $m_{0}>m_{f}, m_{t}$ will descend to $m_{f}$ as the iteration accomplishes the maximum number of iteration with the notation maxNI. Since $0 \leq \mu_{i j} \leq 1$, in FLVQ also follows $0 \leq h_{i j} \leq 1$.

The inhibition function $f(t)$ is a positive strict monotone increasing function of $t$, which is used to control the degree of inhibition within the neural lateral interaction. The function $f(t)$ can determine the decreasing rate from fuzzy soft competitive learning to crisp learning (i.e., WTA). For example, using $f(t)=t^{2}$ will have a more strenuous inhibition (small excited states) than using $f(t)=t$, and hence, the use of $f(t)=t^{2}$ will have a faster decreasing rate than the use of $f(t)=$ $t$. In general, $f(t)=t^{2}$ is used. The batch version of FSLVQ denoted by FSLVQ can be constructed on the same construction of batch SOM and can help us to visualize the convergence state and speed up the learning rate. Based on the FCM membership function, the neural lateral interaction function in FSLVQ is defined as follows:

$$
h_{i j}=\left(\frac{\mu_{i j}}{\max _{1 \leq j \leq c}\left(\mu_{i j}\right)}\right)^{1+\frac{f_{t}}{c}}
$$

and the prototype update equation is:

$v_{i}=\frac{\sum_{j} h_{i j} x_{j}}{\sum_{j} h_{i j}}$

Thus the FSLVQ algorithm is as follows:

S1: Fix $c, T$ and give $\varepsilon>0$

S2: Initialize the weights $v_{i}(0), \mathrm{i}=1, \ldots, c$ Specify $f(t)=t^{1 / 2}, m=2$ and $\operatorname{maxNI}$. Input all feature vectors $x_{j}, j=1, \ldots, n$ Set the iteration counter $t=1$.

S3: For $t=1,2, \ldots, T$;

Calculate $\mu_{i j}(t)$ according to $v_{i}(t-1)$ using equation (4), calculate all $h_{i j}$ at time $t$ according to $\mu_{i j}(t)$ using eq.(18), and calculate $v_{i}$ using eq. (19)

S4: Compute

$$
E_{t}=\|v(t)-v(t-1)\|=\sum_{i=1}^{c}\left\|v_{i}(t)-v_{i}(t-1)\right\|
$$

S5: If $E_{t} \leq \varepsilon$, STOP; ELSE next $t$ GOTO S3

\section{PERFORMANCE EVALUATION}

To verify the performance of the segmentation results of the algorithms, the following image quality indices have been used.

\subsection{Mean Squared Error (MSE)}

The MSE often refer to the error signal which is the difference between the original and the test image (segmented image) signals. If one of the signals is an original signal of acceptable quality, and the other is a segmented image of it whose quality is being evaluated, then MSE may also be regarded as a measure of image quality. The MSE is defined as [15]:

$$
\operatorname{MSE}(x, y)=\frac{1}{N} \sum_{i=1}^{N}\left(x_{i}-y_{i}\right)
$$

Where, $x=\left\{x_{i} \mid i=1,2 \ldots N\right\}$, and $y=\left\{y_{i} \mid i=1,2 \ldots N\right\}$ are the original and the test image (segmented image) signals, respectively and $\mathrm{N}$ is the total number of patterns/pixels.

MSE is used not only to evaluate, but also to optimize a large variety of algorithms for images such as medical pattern recognition. Minimizing MSE is a key criterion in selection estimators.

\subsection{Image Quality Index (IQI)}

The principle behind the image quality metrics is that the main function of the human eyes is to extract structural information from the viewing field, and the human visual system is highly adapted for this purpose. Hence, a measurement of structural distortion should be a good approximation of perceived image distortion. Large errors do not always result in large structural distortions. IQI is a relatively new universal objective image quality index which 
measures the structural distortion rather than the error and is easy to calculate and applicable to various image processing applications. The image quality index (IQI) is defined as [16]:

$$
Q=\frac{4 \sigma_{x y} \overline{x y}}{\left({\sigma_{x}}^{2}+{\sigma_{y}}^{2}\right)\left[(\bar{x})^{2}+(\bar{y})^{2}\right]},
$$

Where, $x=\left\{x_{i} \mid i=1,2 \ldots N\right\}$, and $y=\left\{y_{i} \mid i=1,2 \ldots N\right\}$ are the original and the test image (segmented image) signals, respectively and $\mathrm{N}$ is the total number of patterns/pixels.

$$
\begin{aligned}
& \bar{x}=\frac{1}{N} \sum_{i=1}^{N} x_{i}, \quad \bar{y}=\frac{1}{N} \sum_{i=1}^{N} y_{i} \\
& \sigma_{x}^{2}=\frac{1}{N-1} \sum_{i=1}^{N}\left(x_{i}-\bar{x}\right)^{2}, \\
& \sigma_{y}^{2}=\frac{1}{N-1} \sum_{i=1}^{N}\left(y_{i}-\bar{y}\right)^{2}, \\
& \sigma_{x y}=\frac{1}{N-1} \sum_{i=1}^{N}\left(x_{i}-\bar{x}\right)\left(y_{i}-\bar{y}\right)
\end{aligned}
$$

Maximizing IQI is a key criterion in selection estimators. The best value 1 is achieved if and only if $y_{i}=x_{i}$ for all $i=$ $1,2,3 \ldots \mathrm{N}$. The lowest value of -1 occurs when $y_{i}=2 \bar{x}-x_{i}$ for all $\mathrm{i}=1,2,3 \ldots N$. From the literature it is found that MSE is more sensitive to the energy of errors instead of structural distortions as compared to IQI.

\section{EXPERIMENTAL RESULTS AND DISCUSSION}

The clustering algorithms viz., FCM, KCL, FKCL, FSKCL, LVQ, FLVQ and FSLVQ are applied to the Mets Brain MR images. In order to examine and compare the performance and accuracy of segmentation of these clustering algorithms, the following criteria are used: (i) a random initialization to set as cluster prototypes, (ii) set $k=2, m=1.56$, $\varepsilon=0.001, m_{0}=5, m_{f}=3$ and $f_{t}=t^{\wedge} 2$ and (iii) for each setup, the algorithms are run 10 times and the best case according to the value of the IQI and MSE indices has been tabulated. The figures $1(a-h), \quad 2(a-h), \quad 3(a-h)$ and $4(a-h)$ shows the segmentation results of the algorithms and the table 1 and table 2 shows the comparison of the algorithms according to the best case value of the IQ and MSE. The CPU time of the algorithms and the value of the objective function of the algorithms are also compared and are reported in table 3 . It is seen from the figures 1-4 and the tables 1-3 that the fuzzy soft type competitive learning algorithms viz., the FSLVQ and the FSKCL algorithms have better segmentation quality for the MR image segmentation according to the value of IQI and MSE as compared to the FCM, KCL, FKCL, LVQ and FLVQ algorithms for the Mets Brain MR data set. Further, fuzzy type competitive learning algorithm shows better detection of the abnormal tissues in the MR image segmentation as compared to the other competitive learning algorithms viz., the FLVQ and the FKCL segmentation algorithm have better detection of abnormal tissues in MR image as compared to LVQ and KCL algorithm. However, the FCM segmentation has better performance than the KCL segmentation according to the values of IQI and MSE. In the soft type competitive learning algorithm FSLVQ performs better in detection of abnormal tissues in MR image segmentation and thus we can conclude that LVQ based algorithms has better quality MR image segmentation as compared to the KCL based algorithms. The KCL algorithm has poor performance according to the value of IQI, MSE, objective function and the required CPU time as compared to the other algorithms viz., FCM, LVQ, FKCL, FLVQ, FSKCL and FSLVQ. However the FSLVQ requires slightly larger CPU time than the FSKCL and also the FSLVQ algorithm has higher value of objective function than the FSKCL algorithm as shown in tables 1-3.

Table 1: IQI value for the segmentation results of the clustering algorithms for the Mets Brain MR images (entries in bold are the optimal values)

\begin{tabular}{|l|l|l|l|l|}
\hline Algorithm & T1 & T2 & PD & D \\
\hline FCM & 0.5823 & 0.6332 & 0.4802 & 0.4993 \\
\hline KCL & 0.5776 & 0.6212 & 0.4771 & 0.4972 \\
\hline LVQ & 0.6037 & 0.6475 & 0.5272 & 0.5025 \\
\hline FKCL & 0.6210 & 0.6493 & 0.5359 & 0.5221 \\
\hline FLVQ & 0.6288 & 0.6582 & 0.7761 & 0.5276 \\
\hline FSKCL & 0.7173 & 0.6826 & 0.8688 & 0.5694 \\
\hline FSLVQ & $\mathbf{0 . 7 2 1 3}$ & $\mathbf{0 . 7 8 5 2}$ & $\mathbf{0 . 8 8 0 6}$ & $\mathbf{0 . 6 0 9 6}$ \\
\hline
\end{tabular}

Table 2: MSE value for the segmentation results of the clustering algorithms for the Mets Brain MR images (entries in bold are the optimal values)

\begin{tabular}{|l|l|l|l|l|}
\hline Algorithm & T1 & T2 & PD & $\mathrm{D}$ \\
\hline FCM & 0.1540 & 0.1040 & 0.2315 & 0.1638 \\
\hline KCL & 0.1699 & 0.1096 & 0.2330 & 0.1973 \\
\hline LVQ & 0.1479 & 0.1002 & 0.1987 & 0.1630 \\
\hline FKCL & 0.1267 & 0.0946 & 0.1959 & 0.1558 \\
\hline FLVQ & 0.1213 & 0.0942 & 0.0530 & 0.1443 \\
\hline FSKCL & 0.0782 & 0.0450 & 0.0225 & 0.0895 \\
\hline FSLVQ & $\mathbf{0 . 0 7 6 3}$ & $\mathbf{0 . 0 4 2 1}$ & $\mathbf{0 . 0 1 9 7}$ & $\mathbf{0 . 0 7 0 1}$ \\
\hline
\end{tabular}


Table 3: Objective function value and the elapsed time (in sec.) of the clustering algorithms for the Mets Brain MR images (entries in bold are the optimal values)

\begin{tabular}{|l|l|l|l|l|l|l|l|l|}
\hline \multirow{2}{*}{$\begin{array}{l}\text { Of } \\
\text { T1 }\end{array}$} & $\begin{array}{l}\text { Objective } \\
\text { function/Elap } \\
\text { sed time }\end{array}$ & FCM & KCL & LVQ & FKCL & FLVQ & FSKCL & FSLVQ \\
\hline & $\begin{array}{l}\text { Objective } \\
\text { function } \\
\text { Time }\end{array}$ & 43.5085 & 213.5501 & 193.9079 & 20.7141 & 92.3282 & $\mathbf{1 3 . 4 7 6 4}$ & 96.4234 \\
\hline \multirow{2}{*}{ T2 } & $\begin{array}{l}\text { Objective } \\
\text { function }\end{array}$ & 44.7829 & 213.7598 & 199.8660 & $\mathbf{1 3 . 0 7 7 6}$ & 84.7646 & 13.8572 & 102.4075 \\
\cline { 2 - 9 } & $\begin{array}{l}\text { Elapsed } \\
\text { Time }\end{array}$ & 2.230651 & 48.802976 & 45.903368 & 18.939177 & 10.480856 & $\mathbf{1 . 1 7 5 8 8 5}$ & 5.341624 \\
\hline \multirow{2}{*}{ PD } & $\begin{array}{l}\text { Objective } \\
\text { function }\end{array}$ & 47.8858 & 247.1701 & 231.8352 & $\mathbf{1 3 . 1 4 3 4}$ & 97.3379 & 15.3058 & 106.4648 \\
\cline { 2 - 9 } & $\begin{array}{l}\text { Elapsed } \\
\text { Time }\end{array}$ & 10.359184 & 65.989811 & 71.394975 & 21.639937 & 3.978729 & $\mathbf{1 . 1 7 1 4 4 9}$ & 5.314283 \\
\hline
\end{tabular}

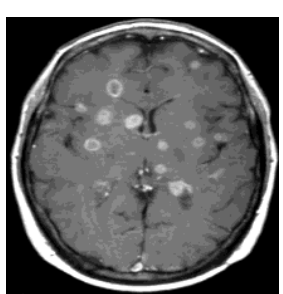

(a)

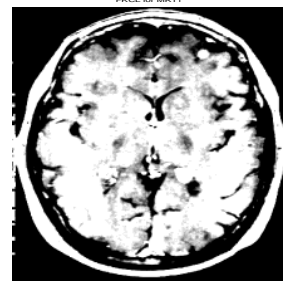

(e)

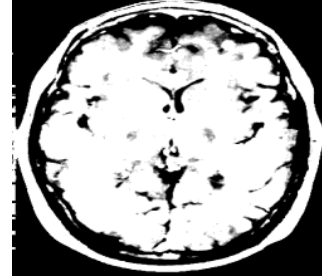

(b)

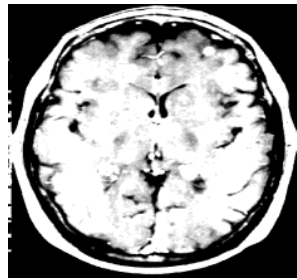

(f)

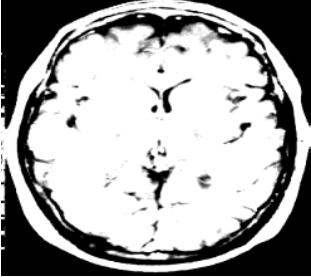

(c)

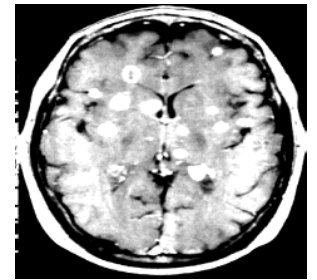

(g)

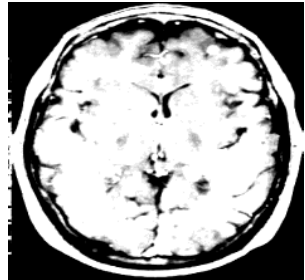

(d)

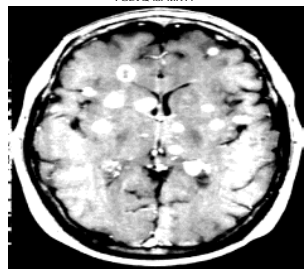

(h)

Fig 1: Segmentation results for the Metsbrain MR T1 data set (a) Original image (b) FCM segmentation, (c) KCL segmentation, (d) LVQ segmentation, (e) FKCL segmentation, (f) FLVQ segmentation, (g) FSKCL segmentation and (h) FSLVQ segmentation. 


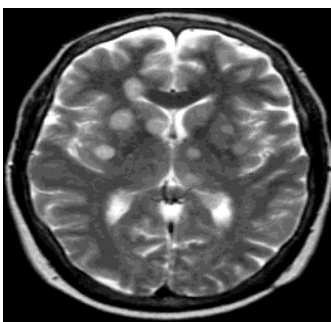

(a)

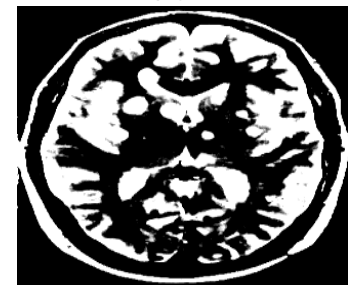

(e)

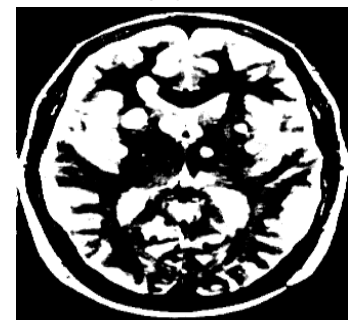

(b)

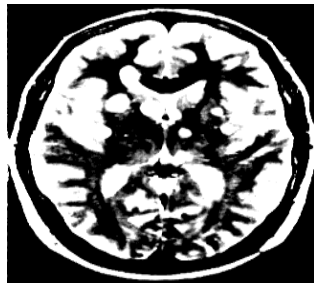

(f)

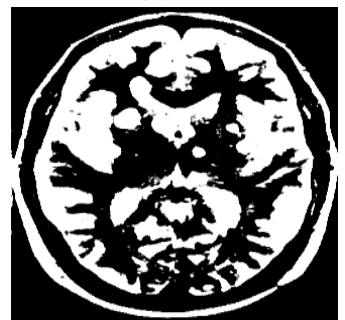

(c)

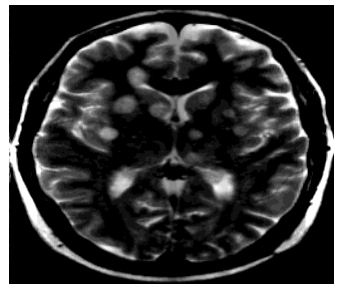

(g)

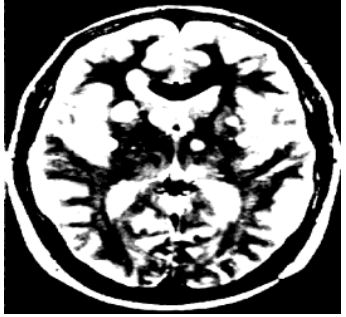

(d)

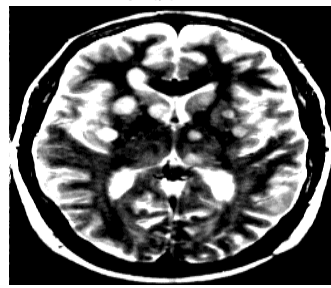

(h)

Fig 2: Segmentation results for the Metsbrain MR T2 data set (a) Original image (b) FCM segmentation, (c) KCL segmentation, (d) LVQ segmentation, (e) FKCL segmentation, (f) FLVQ segmentation, (g) FSKCL segmentation and (h) FSLVQ segmentation.

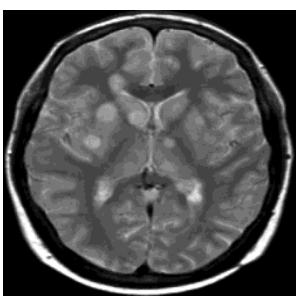

(a)

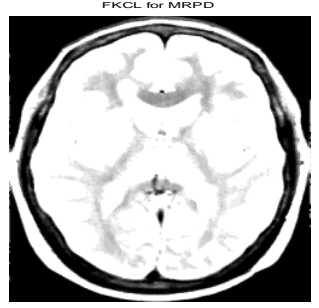

(e)

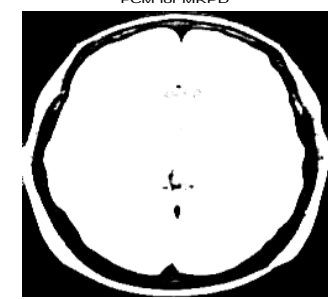

(b)

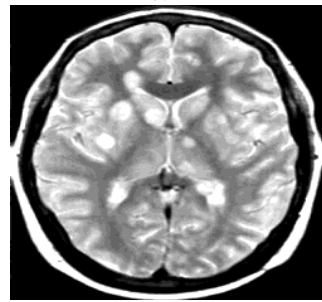

(f)

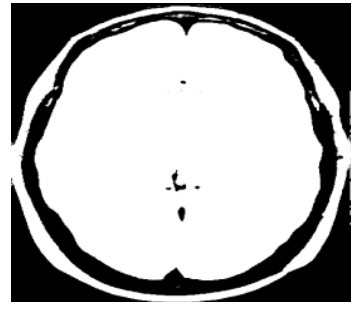

(c)

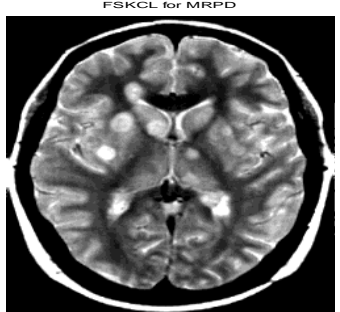

(g)

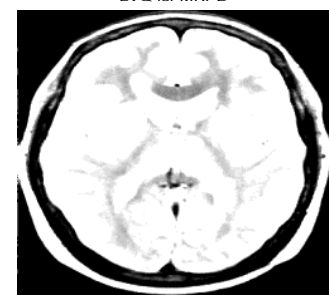

(d)

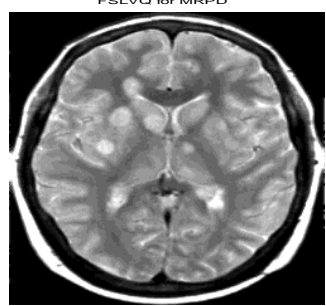

(h)

Fig 3: Segmentation results for the Metsbrain MR PD data set (a) Original image (b) FCM segmentation, (c) KCL segmentation, (d) LVQ segmentation, (e) FKCL segmentation, (f) FLVQ segmentation, (g) FSKCL segmentation and (h) FSLVQ segmentation.

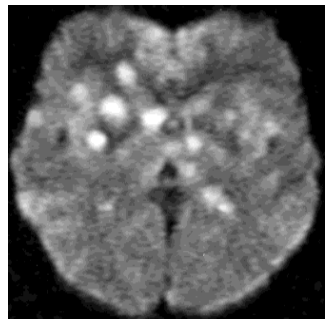

(a)
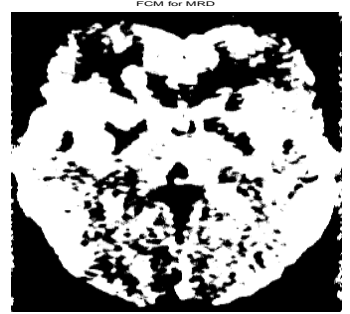

(b)
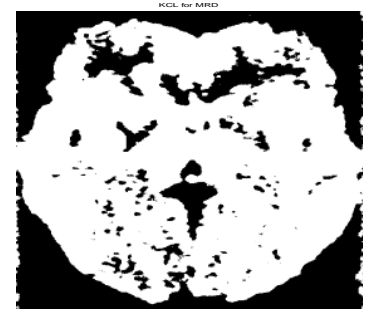

(c)

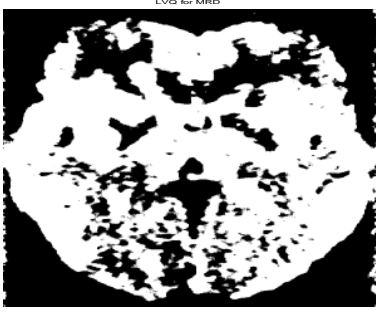

(d) 


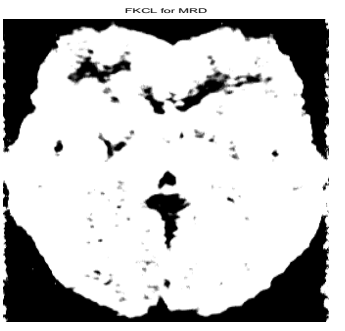

(e)

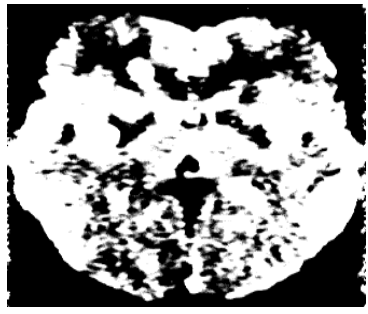

(f)

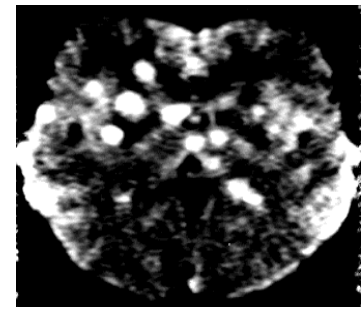

(g)

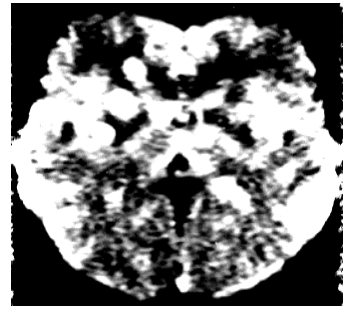

(h)

Fig 4: Segmentation results for the Metsbrain MR D data set (a) Original image (b) FCM segmentation, (c) KCL segmentation, (d) LVQ segmentation, (e) FKCL segmentation, (f) FLVQ segmentation, (g) FSKCL segmentation and (h) FSLVQ segmentation.

\section{CONCLUSION}

Medical imaging process plays a vital role in early diagnosis. Applying the fuzzy pattern recognition diagnosis methods in medical imaging can solve the problems in traditional methods. Various researchers have attempted to introduce different algorithmic approaches for fuzzy pattern recognition that can contribute to the medical diagnosis and prognosis and also for further development of new methods. Fuzzy pattern recognition with an application to medical diagnosis for recognizing the disease in early stage is a challenging research area from both theory and practical point of view. In this paper, a comparative study on the fuzzy competitive learning algorithms for MR image segmentation including the generalized Kohonen's competitive learning (GKCL)-based algorithms (KCL, fuzzy KCL (FKCL), fuzzy soft KCL (FSKCL)) and the learning vector quantization (LVQ)-based algorithms (LVQ, fuzzy LVQ (FLVQ), fuzzy soft LVQ (FSLVQ)) is performed. From the experimental results it is found that the FSLVQ algorithm has better detection of the abnormal tissues in MR image segmentation with the Mets brain data set. However, it has slightly larger CPU time and value of objective function as compared to the FSKCL algorithm.

Moreover, further research across different types of patient problems is needed before all the issues can be addressed and the performance of the FSLVQ algorithm can further be improved in terms of computational CPU time, value of the objective function and the applicability of the algorithm to the real life application domain.

\section{References}

[1] Vipin Tyagi and J. H. Agarwal, "Medical Image Processing Overview", CSI Communications March, 1316 , 2009.

[2] Anke Meyer Base, "Pattern Recognition for Medical imaging", Academic Press, ISBN: 0-12-493290-8, 2004.

[3] James C. Bezdek, James Keller, Raghu Krisnapuram and Nikhil R. Pal, "Fuzzy Models and Algorithms for Pattern Recognition and Image Processing", Springer 2005.

[4] Karen Chia-Ren Lin, Miin-Shen Yang, Hsiu-Chih Liu, Jiing-Feng Lirng and Pei-Ning Wang, "Generalized Kohonen's competitive learning algorithms for opthalmological MR image segmentation", Magnetic Resonance Imaging 21, 863-870, 2003.
[5] Miin-Shen yang, Karen Chia-Ren Lin, Hsui-Chih Liu and Jiing-Feng Lirng, "Magnetic resonance imaging segmentation techniques using batch-type learning vector quantization algorithms. Magnetic Resonance Imaging 25, 265-277, 2007.

[6] Kuo-Lung Wu and Miin-Shen Yang, "A fuzzy-soft learning vector quantization', Nurocomputing 55, 681697, 2003.

[7] Suel A. Mingoti and Joab O. Lima, "Comparing SOM neural network with Fuzzy c-means, Kmeans and traditional hierarchical clustering algorithms", European Journal of Operational Research 174, 1742-1759, 2006.

[8] T. Huntsberger, P. Ajjimarangsee, "Parallel selforganizing feature maps for unsupervised pattern recognition", Internat. J. Gen. Systems 16, 357-372, 1990.

[9] Torres Angela and Neito J.J., "Review Article, Fuzzy Logic in Medicine and Bioinformatics", Hindawi Publishing Corporation, Journal of Biomedicine and Biotechnology, Vol. 2006, Article ID 91908: 1-7, 2006.

[10] Andrea Baraldi and Palma Blonda, "A Survey of Fuzzy Clustering Algorithms for Pattern Recognition-Part I", IEEE Transaction on Systems, Man, and CyberneticsPart B: Cybernetics Vol. 29 No. 6 December, 778-785, 1999.

[11] Andrea Baraldi and Palma Blonda, "A Survey of Fuzzy Clustering Algorithms for Pattern Recognition-Part II", IEEE Transaction on Systems, Man, and CyberneticsPart B: Cybernetics Vol. 29 No. 6 December, 786-801, 1999.

[12] George J. Klir and Bo Yuan, "Fuzzy sets and fuzzy logic theory and applications", Prentice Hall of India Private Limited New Delhi-110 001, 2002.

[13] John Yen and Reza Langari, "Fuzzy Logic Intelligence, Control and Information", Pearson Education, Inc. 1999.

[14] J. C. Bezdek, "Pattern recognition with fuzzy objective function algorithms", Plenum, New York, 1981.

[15] Zhou Wang and Alan C. Bovik, "Mean squared error: love it or leave it? A new look at signal fidelity measures", IEEE signal processing magazine 98 January, 98-117, 2009

[16] Zhou Wang and Alan C. Bovik, "A universal image quality index", IEEE signal processing letters, vol.-9, no.-3, March, 81-84, 2002. 